\title{
Granulomatous hepatitis due to Bartonella henselae infection in an immunocompetent patient
}

\author{
Thomas R VanderHeyden ${ }^{1 *}$, Sherri L Yong ${ }^{2}$, Edward B Breitschwerdt ${ }^{3}$ Ricardo G Maggi ${ }^{3}$, Amanda R Mihalik, \\ Jorge P Parada ${ }^{4}$ and Claus J Fimmel ${ }^{1}$
}

\begin{abstract}
Background: Bartonella henselae (B. henselae) is considered a rare cause of granulomatous hepatitis. Due to the fastidious growth characteristics of the bacteria, the limited sensitivity of histopathological stains, and the nonspecific histological findings on liver biopsy, the diagnosis of hepatic bartonellosis can be difficult to establish. Furthermore, the optimal treatment of established hepatic bartonellosis remains controversial.

Case presentation: We present a case of hepatic bartonellosis in an immunocompetent woman who presented with right upper quadrant pain and a five $\mathrm{cm}$ right hepatic lobe mass on $\mathrm{CT}$ scan. The patient underwent a right hepatic lobectomy. Surgical pathology revealed florid necrotizing granulomatous hepatitis, favoring an infectious etiology. Despite extensive histological and serological evaluation a definitive diagnosis was not established initially. Thirteen months after initial presentation, hepatic bartonellosis was diagnosed by PCR studies from surgically excised liver tissue. Interestingly, the hepatic granulomas persisted and Bartonella henselae was isolated from the patient's enriched blood culture after several courses of antibiotic therapy.

Conclusion: The diagnosis of hepatic bartonellosis is exceedingly difficult to establish and requires a high degree of clinical suspicion. Recently developed, PCR-based approaches may be required in select patients to make the diagnosis. The optimal antimicrobial therapy for hepatic bartonellosis has not been established, and close follow-up is needed to ensure successful eradication of the infection.
\end{abstract}

Keywords: Granulomatous hepatitis, Bartonella henselae, Diagnosis, Treatment

\section{Background}

Cat scratch disease (CSD) is caused by B. henselae, a gram-negative, aerobic alpha Proteobacterium that is transmitted by the bite or scratch of a cat. The traditional diagnostic criteria for CSD include (1) contact with a cat and history of a scratch or other inoculation event, (2) positive cat scratch skin test reaction, (3) regional lymphadenopathy with no other apparent etiology, and (4) characteristic histopathologic features on biopsy [1]. The development of PCR-based diagnostic assays has revealed a growing number of cases in which the traditional diagnostic criteria for CSD were absent [2].

Hepatic bartonellosis has been reported in 1-2\% of CSD cases, and represents the third most common

\footnotetext{
* Correspondence: tvanderheyden@lumc.edu

'Department of Internal Medicine, Division of Gastroenterology, Hepatology and Nutrition, Loyola University Medical Center, 2160 South First Avenue,

Maywood, IL 60153, USA

Full list of author information is available at the end of the article
}

clinical manifestation after fever and lymphadenopathy. The typical symptoms include right upper quadrant pain, fevers, malaise, weight loss, chills and headaches [3].

Establishing the diagnosis of CSD-induced liver disease can be challenging:

Radiologic evaluation by contrast-enhanced CT may show a range of abnormalities, with lesions that are hypoattenuating, iso-attenuating, or show rim enhancement relative to the uninvolved liver [4]. MRI imaging may show suggestive imaging patterns of the granulomas [5].

Histologically, hepatic bartonellosis is characterized by the presence of necrotizing granulomas. They are caused by the focal accumulation of activated macrophages, with a surrounding rim of lymphocytes and fibroblasts. However, hepatic granulomas are nonspecific and may be associated with a variety of liver diseases [6]. Steiner silver staining, Bartonella-specific immunohistochemical

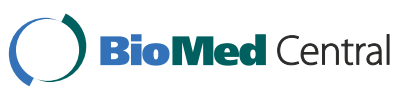


stains, and serologic tests for Bartonella-specific antibodies [7] have been shown to have diagnostic utility but may be less sensitive and specific compared to PCR testing [8-12]. PCR assays do not rely on the presence of a humoral immune response, and may therefore be diagnostic at an earlier stage of infection, or during chronic infection in anergic patients.

Conventional bacterial cultures of liver tissue are rarely diagnostic of B. henselae hepatitis. However, Breitschwerdt and colleagues have recently developed an optimized enrichment culture method that enhances diagnostic detection and molecular typing (by DNA sequencing of the PCR amplicon) of B. henselae and other Bartonella spp. from venous blood. This highly sensitive method is able to detect active infection in patients who lack $B$. henselae antibodies and in whom conventional bacterial cultures have been negative [13,14].

In this study, we describe how the use of optimized serum and tissue PCR assays resulted in the establishment of $B$. henselae hepatitis in a young, immunocompetent patient with severe granulomatous hepatitis.

\section{Case presentation}

In August, 2008, a 36 year-old woman with no prior history of liver disease presented to her primary care physician complaining of abdominal pain of five days duration. The pain was constant, sharp, localized to the right upper quadrant, non-radiating, and associated with nausea but no vomiting. Review of systems was positive for fatigue. Initial laboratory testing revealed only a mildly elevated alanine aminotransferase (ALT) of $42 \mathrm{IU} / \mathrm{L}$. A right upper quadrant ultrasound demonstrated hepatomegaly and steatosis. Two weeks later, the patient presented to an outside hospital with worsening right upper quadrant pain, low-grade fevers, nausea and vomiting. An MRI of the abdomen revealed a two $\mathrm{cm}$ enhancing lesions of the right hepatic lobe. Fine needle biopsy of the lesion demonstrated a nonspecific, mixed inflammatory cellular infiltrate and steatohepatitis. There was no evidence of malignancy. Fungal and mycobacterial cultures were negative. The patient was discharged without a specific diagnosis, and no treatment was instituted.

Two months later, the patient presented to Loyola University Medical Center with worsening right upper quadrant pain and fever. An abdominal CT scan revealed a 4.8 $\times 4.7 \mathrm{~cm}$ mass lesion involving the right hepatic lobe that was suspicious for malignancy (Figure 1). The patient underwent a partial right hepatectomy with excision of the mass. Histopathological studies of the resection specimen showed florid necrotizing granulomatous inflammation with pseudotumor formation (Figure 2, 3). Immuno-histochemical stains for mycobacteria, fungal organisms, and cytomegalovirus were negative. The patient's presenting

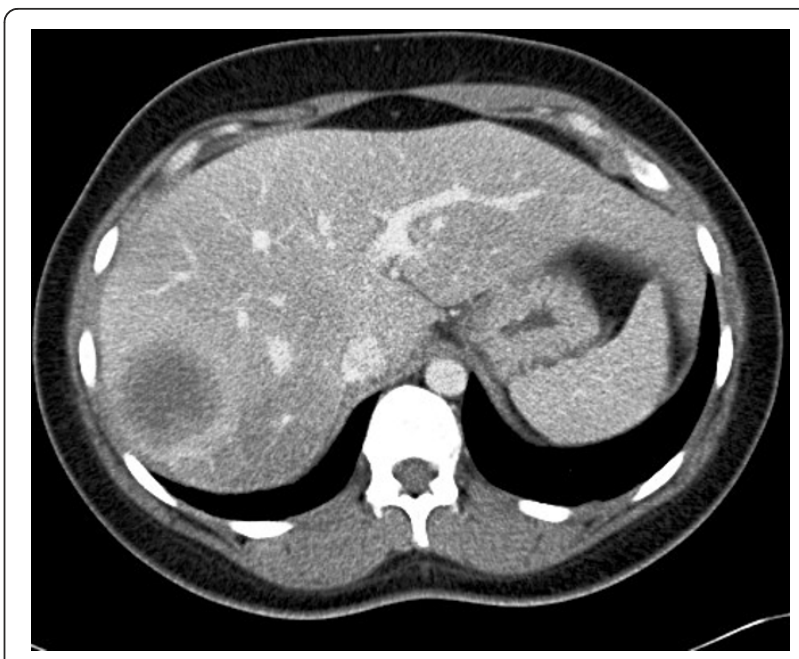

Figure 1 Abdominal CT image demonstrating a large mass lesion in the right hepatic lobe.

symptoms resolved, and she was discharged home after an uneventful postoperative recovery.

In April of 2009, the right upper quadrant pain and low-grade fevers recurred. The patient presented to an outside hospital where an extensive evaluation was initiated (Table 1). Serologies for acute viral hepatitis were negative. Qualitative antibodies for toxoplasmosis, human immunodeficiency virus, and Entamoeba histolytica were negative. An EIA for Borrelia burgdorferi antibodies was negative. IFA assays for Bartonella henselae and Bartonella quintana were negative. Testing for urinary histoplasmosis and blastomycosis antigens and

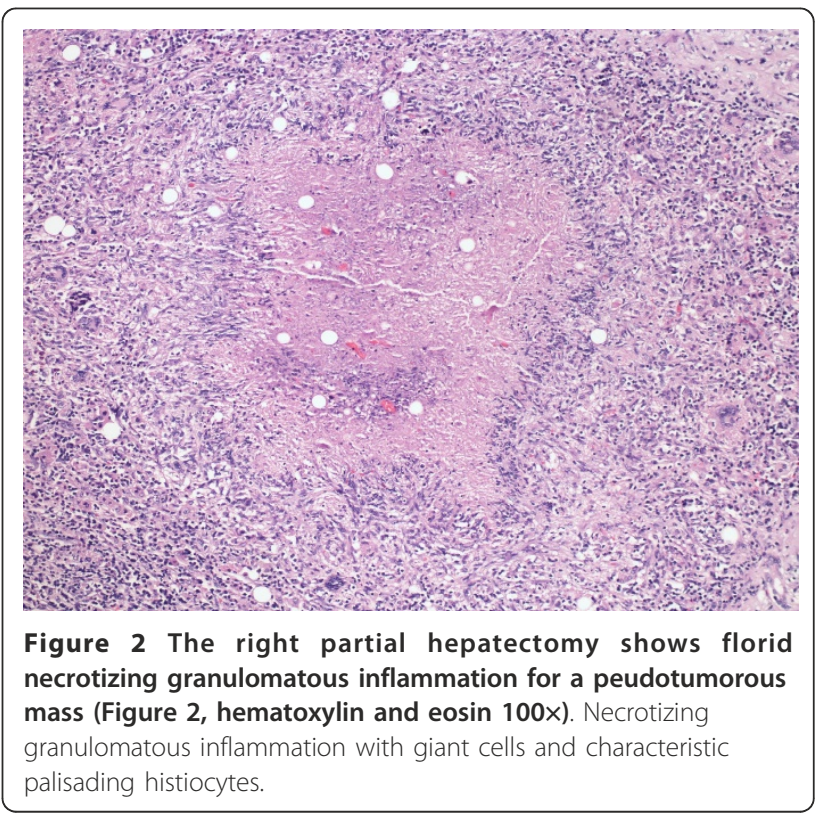




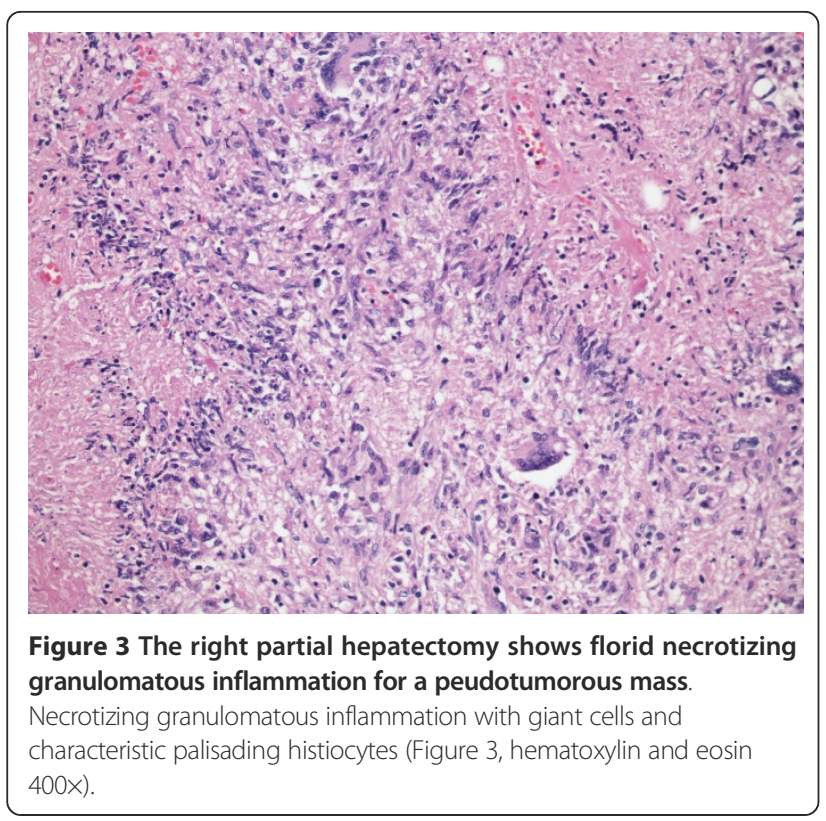

cryptococcus serum antigens were negative. Skin testing for tuberculosis and quantiferon gold were negative. A random liver biopsy showed mild macro- and microvesicular steatosis and non-specific chronic inflammation. Repeat special stains, fungal and mycobacterial cultures were negative. The patient's symptoms improved, and she was discharged home on analgesics.

In July of 2009, the patient once again presented to an outside hospital with right upper quadrant pain, nausea, and fevers of up to $102 .{ }^{4 \circ} \mathrm{F}$. An abdominal CT revealed several low attenuation lesions involving both segments of the liver, with the largest one measuring $4.1 \times 2.9 \mathrm{~cm}$ (Figure 4). The patient was transferred to Loyola University Medical Center for further evaluation. A detailed travel and exposure history to ticks, rodents or other vectors of unusual infectious etiologies was unrevealing. On physical examination, the patient was afebrile. There was no lymphadenopathy or hepato-splenomegaly. Her right upper quadrant was tender to palpation without rebound tenderness or guarding. Laboratory analysis revealed an aspartate aminotransferase (AST) of $93 \mathrm{IU} / \mathrm{L}$ UNITS, alanine aminotransferase (ALT) of $74 \mathrm{IU} / \mathrm{L}$, alkaline phosphatase of $193 \mathrm{IU} / \mathrm{L}$, and total bilirubin of $0.6 \mathrm{mg} / \mathrm{dL}$. The hemoglobin was $13.4 \mathrm{gm} / \mathrm{dL}$, and the WBC count was $4.9 \mathrm{~K} / \mathrm{UL}$ with a normal differential. Bacterial blood cultures, urinalysis, stool cultures, and stool tests for Clostridium difficile were negative. A chest radiograph showed no infiltrates or lymphadenopathy. The antinuclear antibody was 1:40, and anti-mitochondrial antibody testing was negative.

On the third day of her hospitalization, the patient developed a temperature of $101 .{ }^{1 \circ} \mathrm{F}$. A comprehensive

Table 1 Serological tests performed to identify the etiology of granulomatous hepatitis in the patient

\begin{tabular}{|c|c|c|c|}
\hline Test & Reference Range & Result Admission 1 & Result Admission 2 \\
\hline Antibody to human immunodeficiency virus & Negative & Negative & Negative \\
\hline Quatiferon Gold & Negative & Negative & \\
\hline \multicolumn{4}{|l|}{ Epstein Barr virus } \\
\hline $\lg M$ & Negative & Negative & \\
\hline$\underline{\lg G}$ & Negative & Positive & \\
\hline \multicolumn{4}{|l|}{ Bartonella Antibody } \\
\hline B. quintana-lgM, IgG & Negative at 1:20, 1:60 dilution & Negative & Negative \\
\hline B. hensalae & & Negative & \\
\hline Lyme Antibody & Negative & Negative & \\
\hline \multicolumn{4}{|l|}{ Toxoplasma } \\
\hline $\lg M$ & Negative & Negative & \\
\hline $\lg G$ & Negative & Negative & \\
\hline Rapid plasma reagin & Negative & & Negative \\
\hline Entamoeba Antibody & Negative & Negative & \\
\hline Coxiella bruniti (ELISA) & Negative & & Negative \\
\hline \multicolumn{4}{|l|}{ Brucella Antibody } \\
\hline $\lg M$ & Negative at 1:20 dilution & & Negative \\
\hline $\lg G$ & Negative at 1:60 dilution & & Negative \\
\hline Cyrptococcal Ag (serum) & Negative & Negative & \\
\hline Histoplasma Ag (urinary) & Negative & Negative & \\
\hline Blastomycosis Ag (urinary) & Negative & Negative & \\
\hline
\end{tabular}




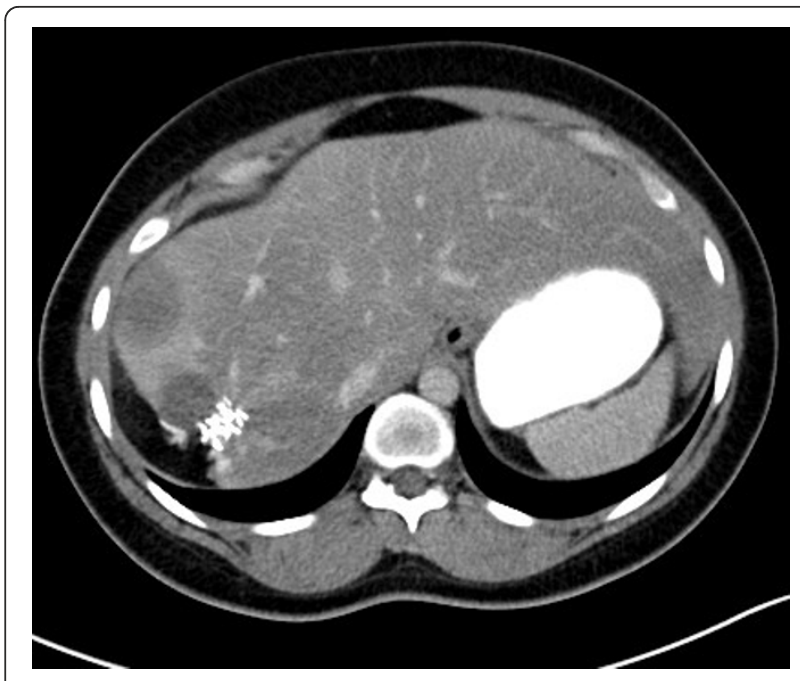

Figure 4 Repeat abdominal CT after initial hepatic resection. Several new, low-attenuation lesions are present in the right hepatic lobe. Similar, smaller lesions were present in the left hepatic lobe (not shown).

investigation for fever of unknown origin was initiated (see Table 1). Antibody titers for Bartonella quintana, and Brucella sp. were negative. An RPR was negative. Repeat HIV antibody testing was negative. A peripheral smear for malaria was negative. A transesophageal echocardiogram did not reveal any valvular vegetations. The patient refused a lumbar puncture. A percutaneous liver biopsy was performed and revealed extensive granulomatous hepatitis with occasional fibrin rings, with a background of mixed, micro- and macro-vesicular steatosis. Based on the presence of fibrin rings, the possibility of Q fever was entertained. However, ELISA testing for Coxiella burnetti antibodies was negative. Repeat special stains and cultures for AFB, fungal organisms, and cytomegalovirus were negative. Steiner silver stains for spirochetes and bacteria - including Bartonella sp.were negative. The patient was empirically treated with a seven-day course of piperacillin and tazobactam. She remained afebrile for the remainder of her hospitalization. Based on the diagnosis of granulomatous hepatitis and the unrevealing workup for infectious organisms, the patient was started on empiric prednisone. Her liver enzymes, which had already been down-trending at the start of prednisone treatment, normalized over the next several weeks. Her symptoms resolved, and she was discharged home on a tapering dose of prednisone.

Based on the striking features of her hepatic granulomas, the possibility of hepatic Bartonella infection was raised, despite the negative Bartonella antibody titers. A sample of formalin-fixed liver tissue from the initial liver resection was sent to the University of Arkansas for PCR testing. Using previously validated assay conditions, a $153 \mathrm{bp}$ fragment of the $B$. henselae $16 \mathrm{~S}$ rRNA gene was amplified, and confirmed by Southern blot hybridization $[15,16]$. Upon further questioning, the patient reported that she had intermittently come in contact with a cat while visiting her mother's house, although she did not recall any cat scratches or bites.

Based on the presumptive diagnosis of hepatic bartonellosis, the patient was started on azithromycin $250 \mathrm{mg}$ daily, and her prednisone was discontinued. Two weeks later, the patient developed diarrhea and abdominal cramping that were attributed to her antibiotic. Azithromycin was discontinued, and the patient was started on a nine-week course of clarithromycin at a dose of 500 mg twice daily. Her symptoms completely resolved.

In May of 2010, the patient presented to our emergency room with recurrent right upper quadrant pain. On examination, she was afebrile and her vital signs were stable. There was no jaundice. Right upper quadrant tenderness was present in the area of the excisional scar. There was no palpable hepato-splenomegaly. No skin rashes were present. AST and ALT levels were elevated to $109 \mathrm{IU} / \mathrm{L}$ and $75 \mathrm{IU} / \mathrm{L}$, respectively. The total bilirubin, alkaline phosphatase, and INR were normal. A liver-protocol CT revealed a $2.4 \mathrm{~cm}$, low-density lesion at the previous surgical site, which was thought to be nonspecific. However, given her recurrent symptoms, she was empirically treated with a six-week course of ciprofloxacin $500 \mathrm{mg}$ twice daily.

At a subsequent clinic visit in September of 2010, she reported improvement in her abdominal pain. However, the AST and ALT activities had risen to $139 \mathrm{IU} / \mathrm{L}$ and 195 IU/L, respectively. A transjugular liver biopsy demonstrated small, scattered, non-caseating granulomas with a background of micro- and macrovesicular steatosis. A sample from the biopsy was sent to the University of Washington for PCR analysis. Using conventional PCR conditions and primers targeting the ribC gene (5'GATATCGGTTGTGTTGAAGA-3', 5'-AATAAAAGGTATAAAACGCT-3') [17], a 393 bp PCR product specific for $B$. henselae was amplified from the biopsy material. In order to determine whether the patient was bacteremic, venous blood samples were sent to the Intracellular Pathogens Research Laboratory (IPRL), Center for Comparative Medicine and Translational Research, College of Veterinary Medicine, North Carolina State University. Using a previous described diagnostic platform [13,14], conventional PCR targeting the 16S-23S intergenic spacer (ITS) region was performed on DNA extracted from blood, serum, and Bartonella alpha Proteobacteria growth medium (BAPGM)enrichment blood cultures. No amplification products were obtained from pre-enrichment blood and serum samples obtained on three sequential days. In contrast, a target band was obtained from one of three post-enrichment blood cultures. DNA sequencing of the amplicon was diagnostic for the ITS region of the SA2 
strain of B. henselae, thereby establishing $B$. henselae bacteremia. The patient's serum tested negative for antibodies against $B$. henselae, B. koehlerae, or Bartonella vinsonii subsp. berkhoffii genotypes I, II or III antigens [14]. Subsequently, using 16S-23S ITS primers, B. henselae DNA (SA2 strain by DNA sequencing) was amplified and sequenced from paraffin blocks containing material from the initial liver resection (January 2009) and from the follow-up biopsy obtained in September of 2010 (Figure 5). These data demonstrated the presence of the SA2 B. henselae strain in the patient's liver throughout her course. Immunohistochemical staining for $B$. henselae using a recently described $B$. henselae monoclonal antibody assay [18] showed rare coccobacilli with positive staining and appropriate morphology for $B$. henselae, however, clusters of organisms were not visualized (data not shown).

\section{Conclusions}

Our case illustrates the unique challenges of establishing and treating $B$. henselae-induced granulomatous hepatitis.

With regard to the establishment of the diagnosis, many of the typical clinical and laboratory features of CSD were absent. Similar to several case reports in the literature, our patient presented with atypical and nonspecific constitutional symptoms [19]. Steiner silver stains of infected liver tissue and immunohistochemical assays did not reveal the culprit organism, and serologic tests failed to detect $B$. henselae-specific antibodies. A high degree of clinical suspicion, persistent attempts to establish a specific diagnosis, and the use of optimized enrichment cultures and PCR amplification were ultimately required to establish the diagnosis in our patient.

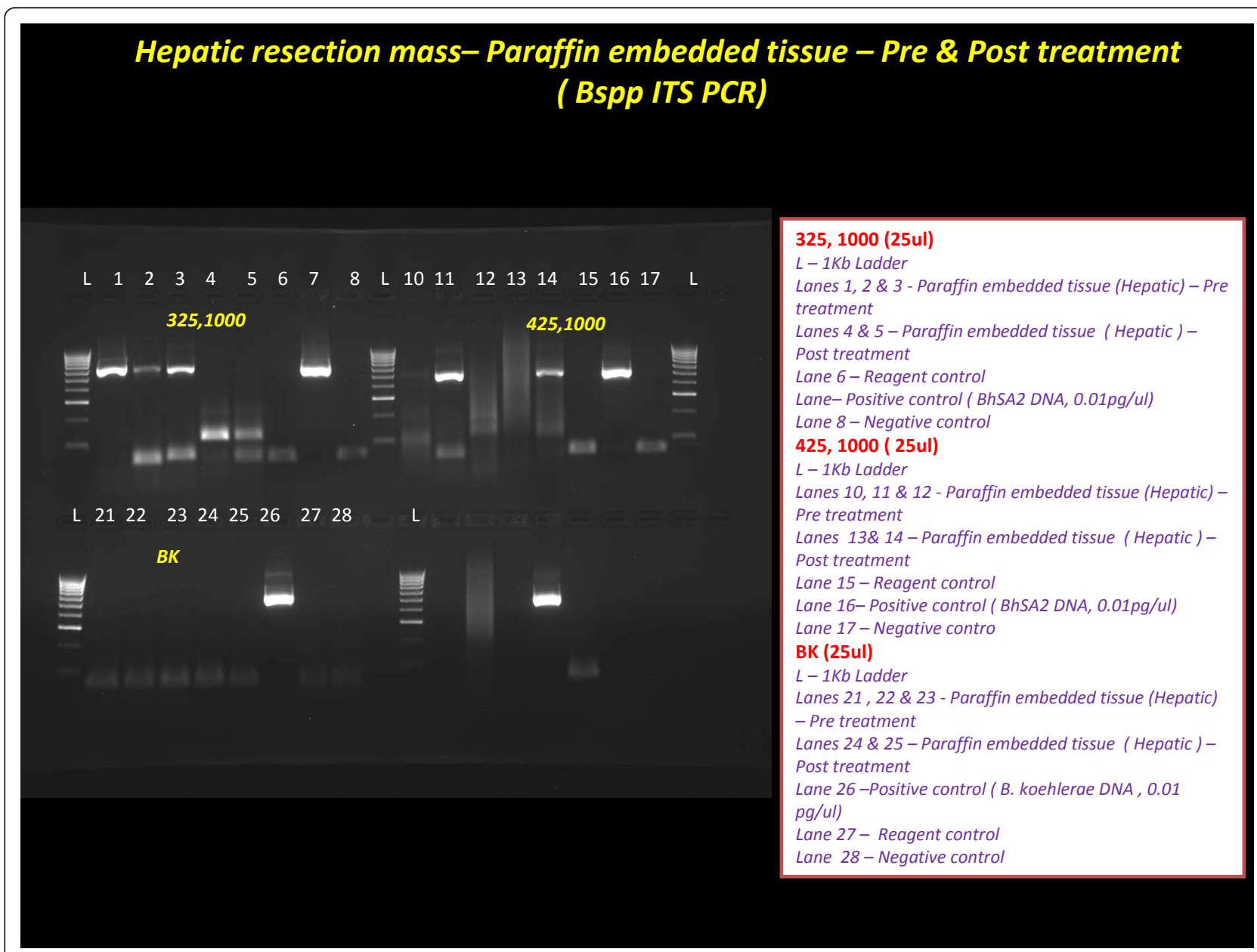

Figure 5 PCR results targeting the Bartonella 16S-23S rRNA intergenic spacer region using primers 325-1000 and primers 425-1100 (top row) and Bartonella koehlerae specific primers (bottom row). With primers 325-1000, amplicons were obtained in lanes 1-3 from the resected liver lobe (pre-antibiotic treatment) but not in Lanes 4-5 (post-treatment liver biopsy). With primers 425-1000, single amplicons were obtained in the pre- (Lane 2) and post-treatment (Lane 5) liver samples. By DNA sequencing, all amplicons corresponded to a 16S-23S strain of B. henselae. No amplicons were obtained with the $B$. koehlerae primer set. $\mathrm{RC}=\mathrm{DNA}$ extraction control, lane, $\mathrm{BH}=\mathrm{B}$. henselae (Houston I strain) $\mathrm{PO}=\mathrm{PCR}$ negative control, $\mathrm{M}=\mathrm{Kb}$ DNA ladder. 
Strikingly, PCR assays targeting three distinct sequences and performed at three different laboratories confirmed the presence of $B$. henselae at multiple time points in the patient's liver and serum, suggesting that this bacterium was the likely causative agent in this case.

With respect to treatment options, our patient's course challenges the traditional view of CSD as an inevitably self-limited disease. Recent guidelines have stressed the importance of prolonged treatment and antibiotic combination therapy in subgroups of patients with CSD, including individuals with HIV infection, bacillary angiomatosis, peliosis hepatis, retinitis, chronic bacteremia, endocarditis, chronic lymphadenopathy and neurological disorders [20]. However, prospective studies validating these recommendations are lacking.

In vitro testing has demonstrated sensitivity of $B$. henselae strains to a wide spectrum of antibiotics including beta-lactams, macrolides, cephalosporins, aminoglycosides, flouroquinolones, doxycycline and rifampin [21]. However, most of these agents are bacteriostatic and fail to eliminate the bacterium when used as monotherapy. In the only prospective, double-blind, placebo-controlled study on immunocompetent patients with CSD [22], azithromycin monotherapy resulted in improved lymphadenopathy but did not prevent dissemination or infectious complications. This disappointing result may have been due to the development of azithromycin resistance by $B$. henselae [23]. Recurrent bacteremia has been described in several reports [24], even after a prolonged antibiotic course.

Our review of the published literature identified a small number of retrospective studies using antibiotic combination therapy: Arisoy and colleagues demonstrated the efficacy of rifampin and gentamycin in a small cohort of pediatric patients [25]. In a single case report, the triple combination of doxycycline, erythromycin and azithromycin cleared B. henselae-induced, post liver-transplant granulomatous hepatitis [26,27]. A combination of doxycycline (100 mg twice daily) and rifampin (300 mg twice daily) was used successfully to treat $B$. henselae retinitis [28]. Prednisone has been suggested as adjunctive therapy in patients with antibioticrefractory hepatic bartonellosis. However, immunosuppression should be used with caution, as it might contribute to the development of Bartonella endocarditis [29,30].

Our patient was initially treated with surgical resection, reminiscent of a previous case report by Murano in which a giant, B. henselae-induced granuloma in a 10-year old child required a partial hepatectomy [31]. This was followed by multiple courses of empiric antibiotic therapy, including piperacillin/tazobactam, azithromycin, clarithromycin, and ciprofloxacin. None of these treatments eradicated the infection. Following the establishment of $B$. henselae bacteremia and the demonstration that $B$. henselae genetic material was present in the patient's liver throughout the course of her disease, we opted to treat the patient with an 8-week course of rifampin (300 mg bid) and doxycycline (100 mg bid). Three months following this most recent treatment, the patient's liver enzymes have completely normalized for the first time, and her constitutional symptoms have not recurred. We are planning on a long-term follow-up and repeat enrichment blood cultures in the future.

\section{Informed consent}

Written informed consent was obtained from the patient for publication of this case report and any accompanying images. A copy of the written consent is available for review by the Editor-in-Chief of this journal.

\section{Acknowledgements}

The authors wish to thank Laura Lamps at the University of Arkansas for performing the 165 rDNA PCR, the Microbiology Department at the University of Washington for performing the RibC PCR, Nandhakumar Balakrishnan at North Carolina State University for performing the 16S-23S ITS PCR, and Dr. Keith Linder at North Carolina State University for the $B$. henselae immunohistochemistry. We also acknowledge Dr. Jonathan Lubens for his critical review of the manuscript.

\section{Author details}

${ }^{1}$ Department of Internal Medicine, Division of Gastroenterology, Hepatology and Nutrition, Loyola University Medical Center, 2160 South First Avenue, Maywood, IL 60153, USA. ²Department of Pathology, Loyola University Medical Center, Maywood, IL 60153, USA. ${ }^{3}$ Intracellular Pathogens Research Laboratory, Center for Comparative Medicine and Translational Research, College of Veterinary Medicine, North Caroline State University, Raleigh, NC 27606, USA. ${ }^{4}$ Division of Infectious Diseases, Loyola University Medical Center, Maywood, IL 60153, USA

\section{Authors' contributions}

TV participated in the clinical care of the patient and wrote the manuscript. SY carried out the pathologic studies. EB participated in the planning of the molecular studies and editing of the final manuscript. RM performed molecular analyses including PCR and DNA sequencing. AM participated in the initial evaluation and treatment of the patient. JP assisted in the selection of anti-microbial therapy. CF served as the primary hepatologist and edited the manuscript. All authors read and approved the final manuscript.

\section{Competing interests}

The authors declare that they have no competing interests.

Received: 27 July 2011 Accepted: 23 January 2012

Published: 23 January 2012

\section{References}

1. Bergmans AM, Groothedde JW, Schellekens JF, et al: Etiology of cat scratch disease: comparison of polymerase chain reaction detection of Bartonella (formely Rochalimaea) and Afipia felis DNA with serology and skin tests. J Infect Dis 1995, 171:916-923.

2. Hansmann $Y$, DeMartino $S$, Piemont $Y$, et al: Diagnosis of cat scratch disease with detection of Bartonella henseale by PCR: a study of patients with lymph node enlargements. J Clin Microbiol 2005, 43:3800-3806.

3. Lamps LW: Hepatic Granulomas with Emphasis on Infectious Causes. Adv Anat Pathol 2008, 15:309-318.

4. Mortele KJ, Segatto E, Ros PR: The infected liver: radiologic-pathologic correlation. Radiographics 2004, 24:937-955. 
5. Dangman BC, Albanese BA, Kacica MA, et al: Cat scratch disease in two children presenting with fever of unknown origin: imaging features and association with a new causative agent, Rochalimaea henselae. Pediatrics 1995, 95:767-771.

6. Drebber U, Kasper HU, Ratering J, et al: Hepatic granulomas: histological and molecular pathological approach to different diagnosis: a study of 442 cases. Liver Int ISSN 2008, 1478:828-834

7. Regnery RL, Olson JG, Perkins BA, Bibb W: Serological response to "Rochalimaea henselae" antigen in suspected cases of cat-scratch disease. Lancet 1992, 339:1443-1445.

8. Dupon M, Savin De Larclause AM, Brouqui $P$, et al: Evaluation of serological response to Bartonella henselae, Bartonella quintana and Afipia felis antigens in 64 patients with suspected cat-scratch disease. Scand J Infect Dis 1996, 28:361-366.

9. Caponetti GC, Pantanowitz L, Marconi S, et al: Evaluation of immunohistochemistry in identifying Bartonella henselae in cat-scratch disease. Am J Clin Pathol 2009, 131:250-256

10. Adivor B, Kletter $Y$, Abulafia $S$, et al: Molecular diagnosis of cat scratch disease: a two-step approach. J Clin Microbiol 1997, 35:1924-1930.

11. Anderson $B$, Sims $K$, Regnery $R$, et al: Detection of Rochalimaea henselae DNA in specimens from cat scratch disease patients by PCR. J Clin Microbiol 1994, 32:942-948.

12. La Scola B, Raoult D: Serological cross-reactions between Bartonella quintana, Bartonella henselae, and Coxiella burnetti. J Clin Microbiol 1996, 34:2270-2274

13. Breitschwerdt EB, Maggi RG, Lantos PM, et al: Bartonella vinsonii subsp. berkhoffii and Bartonella henselae in a father and daughter with neurological disease. Parasites and Vectors 2010, 3:29.

14. Breitschwerdt EB, Maggi RG, Sigmon B, et al: Isolation of Bartonella quintana from a Woman and a Cat following Putative Bite Transmission. J Clin Microbiol 2007, 45:270-272.

15. Anderson B, Sims K, Regnery R, et al: Detection of Rochalimaea henselae DNA in specimens from cat scratch disease patients by PCR. J Clin Microbiol 1994, 32:942-948.

16. Scott MA, McCurley $T L$, Vnencak-Jones $C L$, et al: Cat scratch disease: detection of Bartonella henselae DNA in archival biopsies from patients with clinically, serologically, and histologically defined disease. Am J Pathol 1996, 149:2161-2167.

17. Bereswill S, Hinkelmann S, Kist M, et al: Molecular analysis of riboflavin synthesis genes in Bartonella henselae and use of the ribC gene for differentiation of Bartonella species by PCR. J Clin Microb 1999, 37:3159-3166.

18. Varanat $M$, Broadhurst J, Linder $K$, Maggi M, Breitschwerdt E: Identification of Bartonella henselae in 2 Cats With Pyogranulomatous Myocarditis and Diaphragmatic Myositis. Vet Pathol 2011.

19. Maman E, Bickels J, Ephros M, et al: Musculoskeletal manifestations of cat scratch disease. Clin Infect Dis 2007, 45:1921-1933.

20. Rolain JM, Brouqui P, Koehler JE, et al: Recommendations for treatment of human infections caused by Bartonella species; Minireview. Antimicrobiol Agents Chemother 2004, 48:1921-1933.

21. Dorbecker C, Sander A, Oberle K, et al: In vitro susceptibility of Bartonella species to 17 antimicrobial compounds: comparison of Etest and agar dilution. J Antimicrob Chemother 2006, 58:784-788.

22. Bass JW, Freitas BC, Freitas AC, et al: Prospective randomized double blind placebo-controlled evaluation of azithromycin for treatment of catscratch disease. Pediatr Infect Dis J 1998, 17:447-452.

23. Biswas S, Maggi RG, Papich MG, et al: Comparative activity of pradofloxacin, enrofloxacin and azithromycin against Bartonella henselae isolates derived from cats and a human. J Clin Microbiol 2010, 48:617-618.

24. Sykes JE, Lindsay LL, Maggi RG, et al: Human coinfection with Bartonella henselae and two hemotropic mycoplasma variants resembling Mycoplasma ovis. J Clin Microbiol 2010, 48:3782-3785.

25. Arisoy ES, Correa AG, Wagner ML, et al: Hepatosplenic cat-scratch disease in children: selected clinical features and treatment. Clin Infect Dis 1999, 28:778-784.

26. Bonatti H, Mendez J, Guerrero I, et al: Disseminated Bartonella infection following liver transplantation. Transpl Int 2006, 19:683-687.

27. Thudi KR, Kreikemeier JT, Phillips NJ, et al: Cat scratch disease causing hepatic masses after liver transplant. Liver Int 2007, 27:145-148.
28. Reed JB, Scale DK, Wong MT, et al: Bartonella henselae neuroretinitis in cat scratch disease. Diagnosis, management, and sequelae. Ophthalmol 1998, 105(3):459-466

29. Turner JW, Pien BC, Ardoin SA, et al: A man with chest pain and glomerulonephritis. Lancet 2005, 365:2062.

30. Vikram HR, Bacani AK, DeValeria PA, et al: Bivalvular Bartonella henselae prosthetic valve endocarditis. J Clin Microbiol 2007, 45:4081-4084.

31. Murano I, Yoshii H, Kurashige $H$, et al: Giant hepatic granuloma caused by Bartonella henselae. Pediatr Infect Dis J 2001, 20:319-320.

\section{Pre-publication history}

The pre-publication history for this paper can be accessed here: http://www.biomedcentral.com/1471-2334/12/17/prepub

doi:10.1186/1471-2334-12-17

Cite this article as: VanderHeyden et al:: Granulomatous hepatitis due to Bartonella henselae infection in an immunocompetent patient. BMC Infectious Diseases 2012 12:17.

\section{Submit your next manuscript to BioMed Central and take full advantage of:}

- Convenient online submission

- Thorough peer review

- No space constraints or color figure charges

- Immediate publication on acceptance

- Inclusion in PubMed, CAS, Scopus and Google Scholar

- Research which is freely available for redistribution

Submit your manuscript at www.biomedcentral.com/submit
C Biomed Central 